\title{
The CLAS12 Spectrometer in Hall B at Jefferson Laboratory
}

\author{
Editors: Daniel S. Carman, Volker Burkert, Latifa Elouadrhiri (JLab)
}

Quantum Chromodynamics (QCD) is the theory of strong interactions between quarks and gluons, the fundamental particles that make up composite hadrons such as protons, neutrons, and pions. The laws of QCD are elegant and concise, however, applying them in experimental processes to unravel the structural complexity of hadrons in terms of their fundamental constituents remains one of the most challenging problems in hadronic and nuclear physics today. This challenge motivates the newest generation of facilities and experiments.

In the period from 1997 to 2012 the CEBAF Large Acceptance Spectrometer (CLAS) in Hall B at Jefferson Laboratory was used for studies of inclusive, semi-inclusive, and exclusive reactions from a fixed target with beams of electrons and photons at energies up to $6 \mathrm{GeV}$. CLAS completed the first generation of experiments with tremendous achievements in the field of hadronic physics. CLAS delivered on its initial science program, pioneered new measurements, and opened up new directions in our field. These successes of the past decades have posed and sharpened questions that are central to our understanding of QCD that are now within the reach of the Jefferson Lab $12 \mathrm{GeV}$ energy upgrade.

As part of the Jefferson Laboratory upgrade project that doubled the maximum energy of the electron accelerator to $12 \mathrm{GeV}$, the CLAS spectrometer was decommissioned and the new CLAS12 large acceptance spectrometer was designed, prototyped, built, installed, and commissioned in Hall B in the context of an international collaboration. This new spectrometer will be used for an expansive program of physics topics, including femtographic imaging of the quark distributions with the nucleon, investigations of the spectrum and structure of excited baryons, searches for exotic meson and baryon configurations, and studies of nucleon correlations within nuclei. The experimental program will be carried out with polarized electron beams of energies up to $11 \mathrm{GeV}$ on both unpolarized and polarized hydrogen and deuterium targets, as well as nuclear targets, at beam-target luminosities of up to $1 \times 10^{35} \mathrm{~cm}^{-2} \mathrm{~s}^{-1}$ per target nucleon. This program of experiments requires a broad kinematic coverage in invariant energy $W$ up to $4 \mathrm{GeV}$, four-momentum transfer $Q^{2}$ from $0.05 \mathrm{GeV}^{2}$ to $12 \mathrm{GeV}^{2}$, and nearly complete angular coverage of the final state reaction phase space. The design and construction of CLAS12 is based on the latest state-of-the-art advances in detector and superconducting magnets technologies and associated electronics, which allows for complex event selections at the trigger level and high-rate acquisition of experimental data. CLAS12 is performing a new generation of experiments, requiring high luminosity and large acceptance, conditions that were technically out of reach before.

This special issue focuses on the new CLAS12 spectrometer and provides an overview of its design and performance in beam. It also includes detailed information on the design, construction, and performance of the individual detector packages and the superconducting torus and solenoid magnets at the heart of the spectrometer. Finally, detailed overviews are provided of the data acquisition and trigger systems, offline reconstruction approaches, and the GEANT4-based detector simulation package. This issue serves as the definitive reference for the CLAS12 spectrometer system in Hall B at Jefferson Laboratory. 